\title{
Fast Fourier Transform in the Spiral Honeycomb Image Algebra
}

\author{
Phil Sheridan \\ Griffith University Qld \\ p.sheridan@griffith.edu.au
}

\author{
David M. Alexander \\ University of Technology \\ Sydney, NSW
}

\author{
Kelly S. Nunn-Clark \\ Charles Sturt University \\ Bathurst, NSW
}

\begin{abstract}
The Fourier transform is one of the most important transformations in image processing. A major component of this influence comes from the ability to implement it efficiently on a digital computer. This paper describes one such efficient implementation and discusses its implications to digital technology as well as biological vision. The significance of the fast Fourier transform (FFT) presented in this paper is that it provides geometrical meaning to the regrouping of a Cooley-Tukey type FFT.
\end{abstract}

Key Words fast Fourier transform, hexagonal lattice, image

\section{Introduction}

The Fourier transformation is one of the most important transformations in image processing in particular and signal processing in general. Its origins date back to 1807 when Jean Baptiste Joseph Fourier defined the notion of representing a function as a trigometric series. Until the invention of the digital computer, the Fourier series was a purely analytic tool. However, with the development of the fast Fourier transform the notion has become a useful computational tool. Today, most of us use fast Fourier transforms everyday without even knowing it as this technology is employed in digital cameras, disc drives and even our cell phones [1].

Since its original inception in the early 1960's, the FFT has undergone a multitude of mutations many of which have resulted in patents. The motivation behind the development of these FFT variants is the commercial advantage gained by achieving rapid creation of a signal and a rapid reconstruction of the original signal from it.

This paper describes another variation of the FFT named SHIA-FFT, which is of type Cooley-Tukey and is particular to a mathematical structure known as Spiral Architecture originally described in [2], and later extended by $\mathrm{He}$ and $\mathrm{Wu}$ [3]-[4]. The SHIA-FFT algorithm employed on an image represented on either a hexagonal lattice or a rectangular lattice builds the Fourier transform and its inverse in incremental steps of low pass frequencies to high pass frequencies. This property has implications to biological vision as well as technology and is discussed in this paper.

Middleton [5], described a fast Fourier Transform, named HIP-FFT, on an image represented on a hexagonal lattice. The paper makes two important contributions to the theory of FFTs: 1) it provides empirical evidence for the quality of the Fourier transform when performed on a hexagonal lattice; 2) it provides the mathematical motivation underpinning the FFT on the hexagonal lattice. SHIA-FFT distinguishes itself from HIP-FFT in one regard. HIP-FFT employs a third coordinate system, as defined in [5], to relate the addresses on the hexagonal lattice to the re-grouping process. The SHIA-FFT achieves this regrouping with the use of a transformation from Spiral Honeycomb Image Algebra.

\section{Spiral Honeycomb Image Algebra (SHIA)}

The distribution of photoreceptors on the primate's retina and the distribution of cells in the human cortex [6]-[7] are highly suggestive of primitive image transformations associated with the vision process. A natural data structure that emerges from geometrical considerations of this distribution, named Spiral Honeycomb Mosaic (SHM), is outlined in detail in [2]. The underlying geometry of the SHM is a hexagonal lattice, with each hexagon having a designated positive integer address. The numbered hexagons form clusters of super-hexagons, of size $7^{\mathrm{n}}$, these self-similar superhexagons tile the plane in a recursively modular manner. A super-hexagon of size $7^{\mathrm{n}}$ and its concomitant addressing scheme is seen in Figure 1a. 


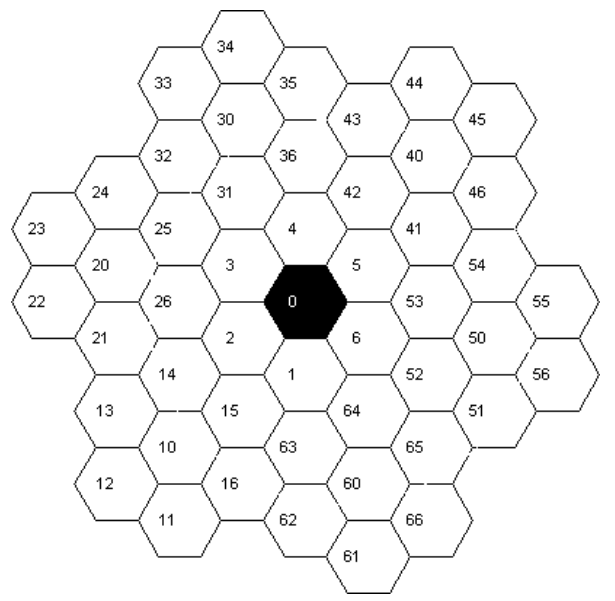

$1 a$.

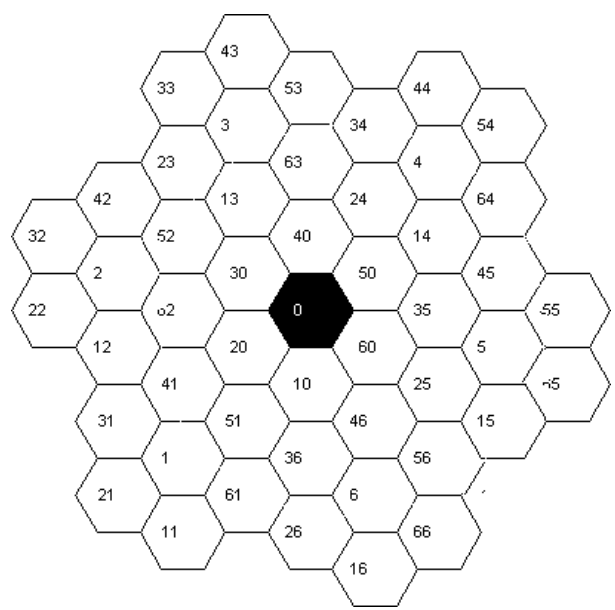

$1 b$.

Figure 1: 1a) displays the address space of a 49 hexagon SHM; 1b) results from applying M10 of SHIA to Figure $1 \mathrm{a}$.

The addressing scheme of the SHM has associated with it a collection of transformations named Spiral Honeycomb Image Algebra (SHIA). These transformations form a powerful mathematical structure known as a Euclidian ring, which possesses almost all the algebraic properties normally attributed to the real and complex number systems. SHIA emerges from the two algebraic operations defined on the SHM, Spiral Addition and Spiral Multiplication. Each of these algebraic operations defines a transformation on the address space of SHM. When a super-hexagon also has associated with it a discrete sampling of a 2-dimensional signal, the algebraic operations take on a geometrical interpretation of the sampled signal. The spatial information embodied in the signal is captured algebraically by the operations. Spiral Addition is associated with translation of the signal and Spiral Multiplication is associated with the dual transformations of rotation and scaling. A critical property of these transformations is that no information of the originally sampled signal is ever lost under the action of the transformations. An explanation for this property and a full discussion of the SHIA is found in [2].

The transformation from SHIA pertinent to the fast Fourier transformation as discussed in this paper is M10 and its inverse. The relation between the address space of Figure 1a and $1 \mathrm{~b}$ is exactly that of M10. Figure 2 (a-d) displays the effect of M10 when applied to an image represented on the SHM. Each of the four images can be derived from successive applications of either M10 or its inverse to any of the other images displayed in the figure. In other words, M10 and its inverse perfectly recover pixel information of the originally sampled image from any of its transformations.

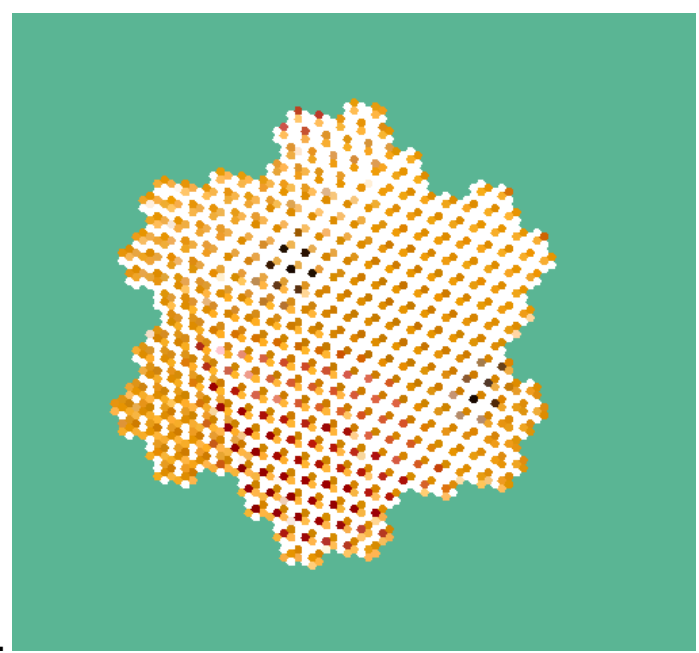

$2 b$.

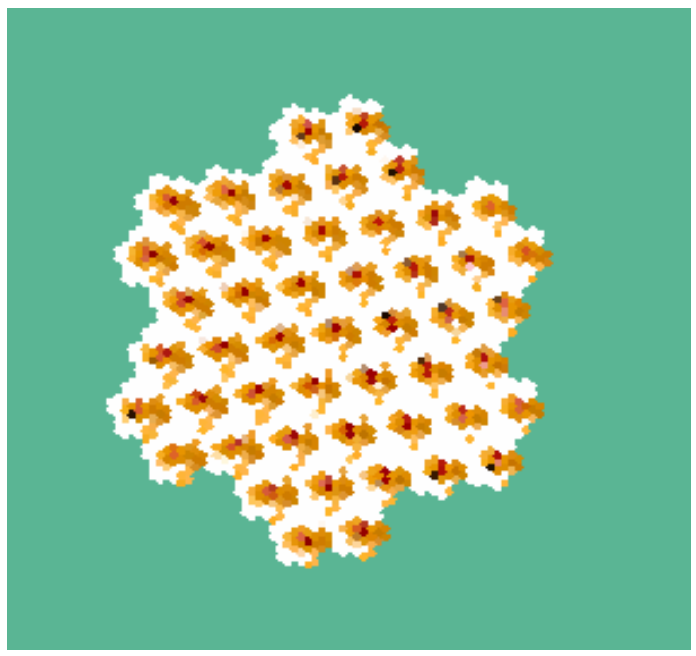




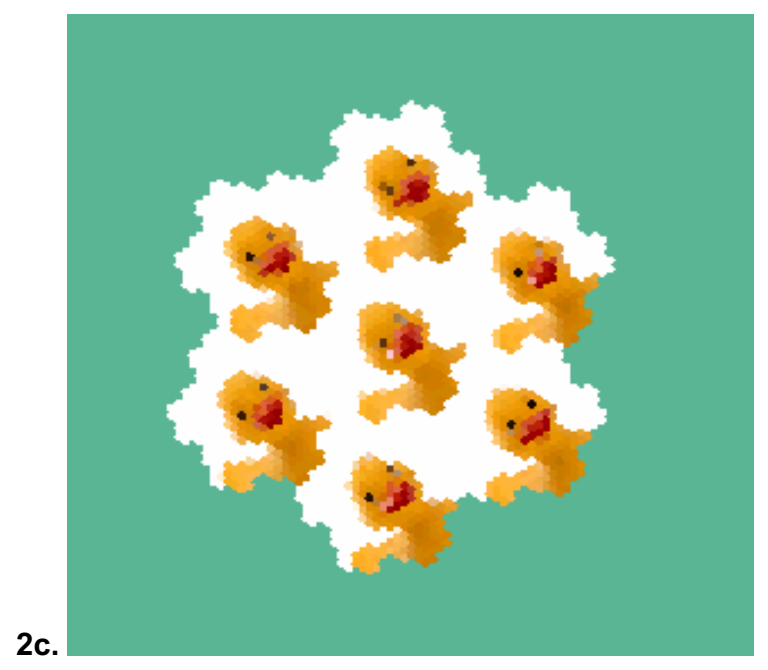

2d.

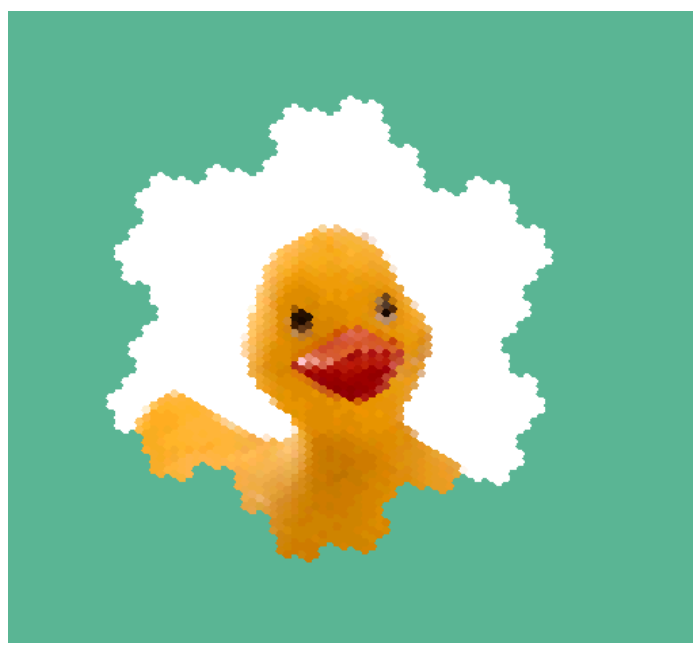

Figure 2. Displays four transformations of an image represented on a SHM of size 2401 pixels. 2a) displays the result of M10 applied to $2 d$; $2 b$ ) displays result of applying M10 to $2 a$; 2c) displays the result of applying M10 to $2 b ; 2 d)$ displays the input image and results from the application of M10 to 2c.

Another property of M10, which is observable in the images displayed in Figure 2, is that each transformation assembles the image at a unique resolution. Figure 2 a represents the image at multiple views of the image at the lowest possible non-trivial resolution. That is, the image is composed of 343 subimages, each of which is composed of seven equally spaced pixels from the input image. If M10 is applied to this image, the resultant image is that displayed in Figure $2 b$, one that is composed of 49 sub-images of size $7^{2}$. Successive applications of M10 reduce the number of sub-images by a factor of seven and increase the size of each sub-image by the same factor.
Once the image of Figure $2 \mathrm{~d}$ is achieved, (one subimage of size $7^{\mathrm{n}}$ ), a further application of M10 results in the image displayed in Figure 2a. In this particular case, the modular aspect of the M10 has produced the identical effect of applying inverse M10 to Figure $2 \mathrm{~b}$.

It is exactly this lossless recovery property and the particular partitioning property of M10 that facilitates the efficient re-grouping of the computations of the fast Fourier transform.

\section{Fast Fourier Transform in SHM}

The fast Fourier transform distinguishes itself from the Fourier transform, commonly known as Discrete Fourier Transform (DFT) by the order in which the computations of the complex arithmetic operations, addition and multiplication, are performed. The DFT is defined as:

$$
\mathrm{F}(\mathrm{u})=\sum_{x=0}^{n-1} f(x) e^{\left(x^{*} u \bmod n\right)} \quad \text { where } \mathrm{u}=(0, \ldots, \mathrm{n}-1)
$$

Where $\mathrm{f}(\mathrm{x})$ represents the input signal as a complex number and $\mathrm{e}^{\left(\mathrm{x}^{*} \mathrm{u} \bmod \mathrm{n}\right)}$ represents the $\left(\mathrm{x}^{*} \mathrm{u} \bmod \mathrm{n}\right)$ address of $n$ equally spaced complex numbers on the unit circle of the complex plane.

A casual analysis of Equation 1 reveals that DFT is of order $\mathrm{O}\left(\mathrm{n}^{2}\right)$ in the number of complex arithmetic operations performed. In contrast, a Cooley-Tukey type fast Fourier transform re-groups the complex operations to achieve an $\mathrm{O}(\mathrm{n} \log (\mathrm{n}))$ algorithm. The SHIA-FFT algorithm is of type Cooley-Tukey and is described informally in Section 3.1. Section 3.2 provides a more formal presentation of the algorithm.

\subsection{Informal Statement of Algorithm}

Initially, the input signal is represented on an SHM of an appropriate size, say $7^{4}$. Denote this collection of values as $\mathrm{f}(\mathrm{x})$. The discrete unit circle of size seven from the Complex plane is mapped onto another SHM. Denote the collection of these values as $\mathrm{u}(\mathrm{x})$. Then a three-step process, localization, discrete Fourier transform and globalisation are applied at each of the $\log (\mathrm{n})$ levels.

Localization is achieved by applying M10 to $\mathrm{f}$. The result is to transform the original spatial domain into that which is observable in Figure 2a. In other words, the effect is a re-grouping of the spatial domain at the lowest resolution. The standard DFT is applied to each of the $7^{(\mathrm{n}-1)}$ sub-images of size 7 . 


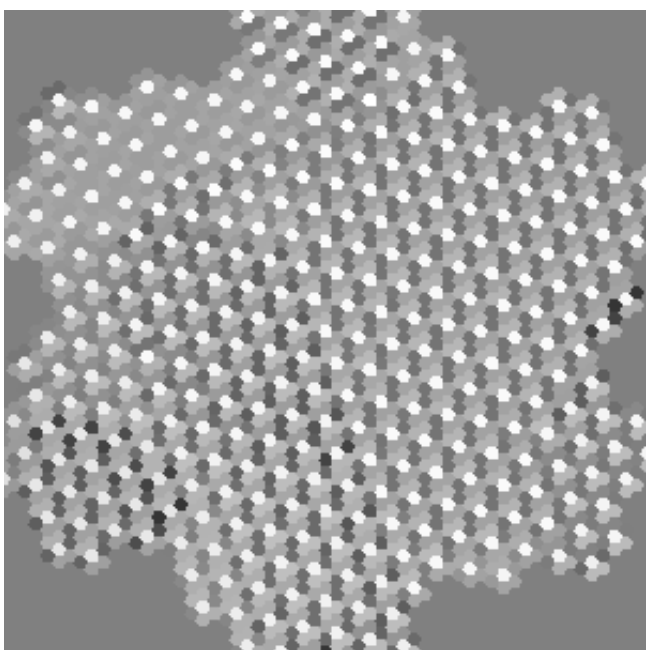

3a. Level 0

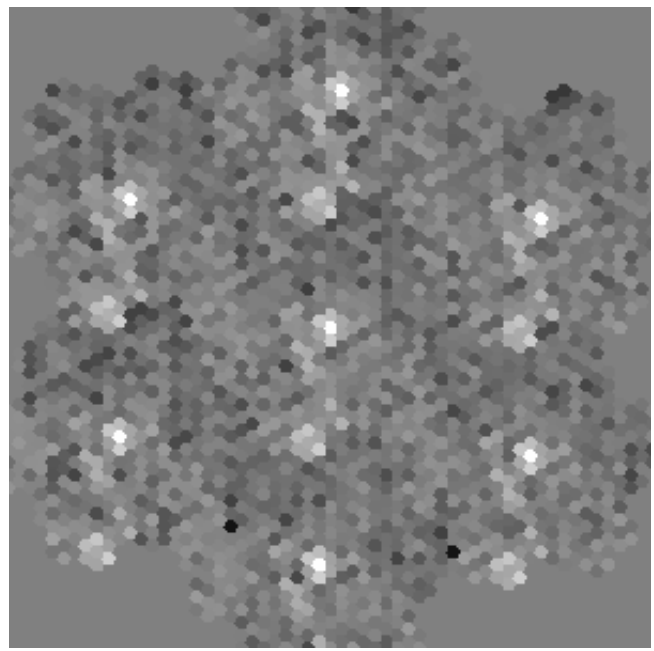

3c. Level 2

Figure 3. Displays the four stages of the Fourier transform of the image displayed in $2 d$.

3a) displays the result on completion of level 0 ;

$3 b)$ displays result on completion of level 1;

3c) displays result on completion of level 2;

3d) displays the completed transform level 3.

The lowest level of the FFT is complete as seen in Figure 3a.

At Level 1, the localization is achieved by applying M10 to both the resulting frequency domain from the previous level and the unit circle of size $7^{2}$. The effect on this transformation on the new spatial domain is a re-grouping, in clusters of seven, at the next higher level of resolution. The effect of M10's application to the new unit circle is a re-grouping, in clusters of seven, of the unit circle into seven clusters, each of which contains seven addresses of equal and maximal

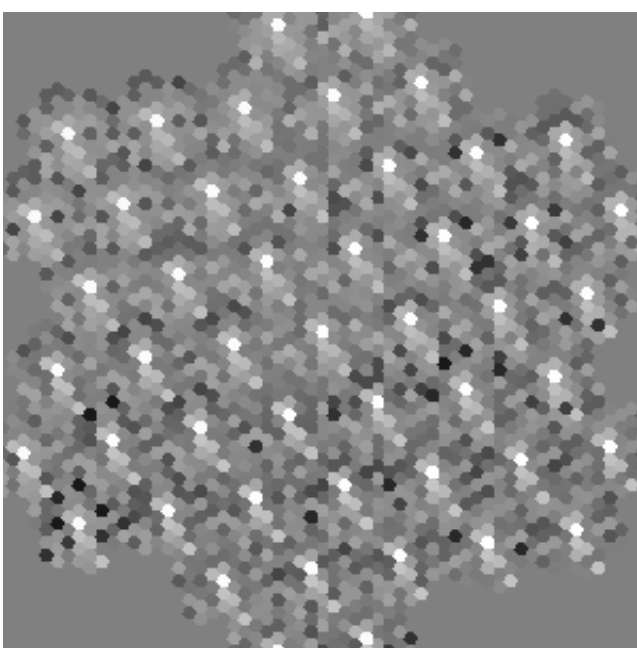

3b. Level 1

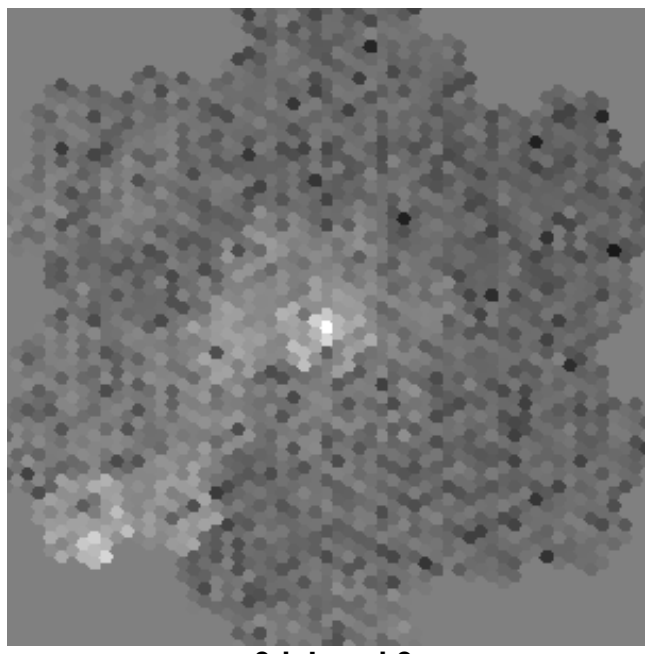

3d. Level 3

spacing on the unit circle. At this stage, the localization process is complete and the DFT on each of the seven clusters is performed. In order to complete the threestep process at this level, the globalisation process is then performed. This amounts to applying the inverse of M10 on each super-hexagon of size 49. The effect of this transform is a re-grouping of the frequency domain into consecutive super-hexagons at the second lowest resolution; sub images of size 49. This result can be seen in Figure 3b.

At each of the subsequent levels, this same threestep process of localization, discrete Fourier transform and globalisation is performed. The results at each level achieve the Fourier transform at the next higher level of resolution, the results of which are presented in Figure $3 \mathrm{c}$ and $3 \mathrm{~d}$.

A formal presentation, in pseudo code, of the process described above follows: 


\subsection{Formal Statement of Algorithm}

DATA:

Integer base $\quad / /$ base of the SHM
Integer imageSize
Integer curSubImageSize $/ /$ current
Integer curNoOfSubImages //current
Array of complex numbers: $\mathrm{f}, \mathrm{F}$, unitCircle, $\mathrm{u}$

START

SET the Input image, as an array of size imageSize

SET curSubImageSize $=1$

SET curNoOfSubImages $=$ imageSize

//initialise spatial domain

INIT $\mathrm{f}$ as an array of size imageSize

/initialise frequency domain

INIT F with the input image

WHILE curSubImageSize $<$ imageSize

curSubImageSize $=$ base $*$ curSubImageSize

curNoOfSubImages $=$ imageSize/curSubImageSize

$\mathrm{f}=$ localiseSpatialDomain $(\mathrm{F}$ )

$\mathrm{F}=$ performDFTLocally $(\mathrm{f})$

$\mathrm{F}=$ globaliseFrequencyDomain $(\mathrm{F})$

RETURN with $\mathrm{F}$

ENDWHILE

END

METHOD localiseSpatialDomain ( F )

CALL M10( F )

RETURN with f

METHOD performDFTLocally ( f ) unitCircle $=$ createUnitCircleSize ( curSubImageSize )

$\mathrm{u}=\mathrm{M} 10$ ( unitCircle)

SET Integer subImage $=0$

WHILE subImage $<$ curNoOfSubImages

Integer address $=$ subImage $*$ curSubImageSize

WHILE (address < curSubImageSize)

dFT( f[ address ] ), u[ address ] )

address $=$ address + base

END WHILE

INCREMENT subImage

END WHILE

RETURN with F

METHOD globaliseFrequencyDomain ( F )

SET Integer address $=0$

SET Integer subImage $=0$

WHILE subImage $<$ curNoOfSubImages

$\mathrm{F}$ [address $]=$ inverseM10 $(\mathrm{F}[$ address $])$

INCREMENT subImage

address $=$ address + curSubImageSize

END WHILE

RETURN with $\mathrm{F}$
METHOD M10( a )

RETURN with a // M10 applied to a

METHOD InverseM10 ( a )

RETURN with a // Inverse M10 applied to a

METHOD createUnitCircleSize ( Integer $\mathrm{n}$ )

RETURN with array of $n$ equally spaced complex numbers from the unit circle

METHOD dFT ( $\mathrm{f}, \mathrm{u}$ )

SET Integer $\mathrm{x}=0$

WHILE $\mathrm{x}<$ base

SET Complex sum $=0$

SET Integer $\mathrm{k}=0$

WHILE $\mathrm{k}<<$ base

$\operatorname{sum}=\operatorname{sum}+(\mathrm{f}[\mathrm{x}] * \mathrm{u}[\mathrm{I} * \mathrm{x} \%$ base $])$

INCREMENT k

$\mathrm{F}[\mathrm{X}]=\operatorname{sum}$

END WHILE

INCREMENT $\mathrm{x}$

END WHILE

\section{Implications of SHMFFT to biological vision and technology}

Although the fast Fourier transform on the SHM as described in this paper, is a $\mathrm{O}(\mathrm{n} \log (\mathrm{n}))$ algorithm, and thereby no more efficient than the other Cooley-Tukey type FFT algorithms, it is significant to researchers in both the image processing and biological vision communities. To the former community, it provides intuitive meaning to what was previously dismissed as just a clever re-grouping of computation in the Fourier Domain. In particular, the fact that the application of M10 at various stages in the production of the frequency domain reveals clearly the relation between the resolution information captured in the frequency domain to each level of the $\log (n)$ levels of computation.

There are implications of M10 to the biological vision community. The M10 transform has been used to describe the patterns of connectivity seen in the primary visual cortex [8]. In particular, the transform describes an isomorphism between the short- and longrange intrinsic connections. The M10 transform has likewise been used to explain the relationship between the retinotopic input mapping of the primary visual cortex and the local mapping of orientation preference and spatial frequency selectivity in the upper layers of the primary visual cortex [8]-[9]. 


\section{Conclusion}

This paper has presented a Cooley-Tukey type fast Fourier transform applied to signals representable on a hexagonal lattice. The key to the algorithm presented is a transformation, M10, from the Spiral Honeycomb Image Algebra. The algorithm is computationally comparable to other FFTs. However, the deeper significance of the algorithm results from the intuitive meaning that it brings to the re-grouping process of the class of FFT algorithms.

\section{References}

[1] Selikoff, S., (2002) "Introduction to the Finite Fourier Transform Including a Brief Review of General Fourier analysis." http://scott.selikoff.net/papers.html

[2] Sheridan, P., Hintz, T. and Alexander, D. (2000) "Pseudo invariant transformations on a hexagonal lattice", Image and Vision Computing, vol. 18, no. 11, pp. 907-917.

[3] He, X., Hintz, T., and Wu, Q. (2002) "Neural Network Based Image Edge Detection within Spiral Architecture", Proc. The International Conference on Image Science, System and Technology, Las Vegas.

[4] Wu, Q. and Reilly, J.P. (1991) "Extension of ESPIRIT Method to Unknown Noise Environments", Proc. International Conference on Acoustics, Speech, and Signal Processing, pp. 3365-3369.

[5] Middleton, L., Sivaswamy, J. (2001) "The FFT in a Hexagonal-image Processing Framework", Proceedings of Image and Vision Computing, New Zealand. pp. 231-236.

[6] Calvin, W.H. (1987) "The brain as a Darwin Machine", Nature 330: pp33-34 (5 November)

[7] Williams, D.R. (1986) "Seeing through the photoreceptor mosaic", Trends in Neuroscience 9, 193-198.

[8] Alexander DM., Sheridan P., Hintz T., and Wright JJ. (2004) "Specification of Cortical Anatomy within the Spiral Harmonic Mosaic Algebra", Proceedings of the 2004 International Conference on Imaging Science, Systems and Technology (CISST'04), p.50-56

[9] Alexander DM., Bourke PD., Sheridan P., Konstandatos O., and Wright JJ. (2004) "Intrinsic connections in tree shrew V1 imply a global to local mapping", Vision Research, 44(9):857-76. 